VIRAL INFECTION

\section{Interfering with HIV infection}

The antiviral cytokine interferon- $\alpha$ (IFNa) has inconsistent effects on HIV infection: some cell types become resistant to infection after IFNa treatment, whereas others remain susceptible. Two studies have identified a new HIV restriction factor, the IFN-inducible myxovirus resistance 2 protein (MX2; also known as MXB), and indicate that it is responsible for these differences.

IFNa induces an antiviral response in cells by upregulating a range of cellular factors, some of which are known

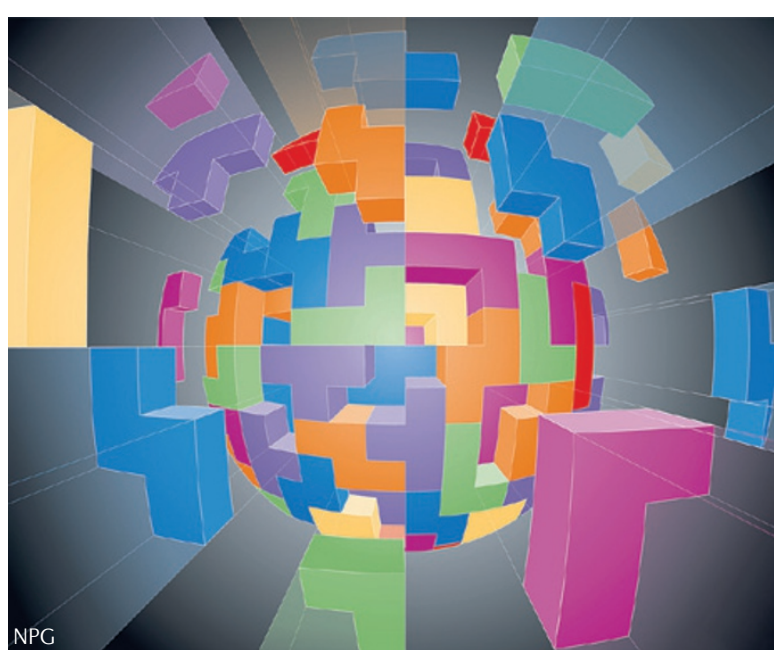

HIV restriction factors. In search of new restriction factors, Goujon et al. screened cell lines and primary cells that had varying levels of IFNa responsiveness (and subsequent HIV resistance) by transcriptional profiling. The authors identified 14 IFNinduced genes that were upregulated in responsive HIV-resistant cells; in ectopic expression experiments only one of these genes, $M X 2$, inhibited HIV infection. Silencing of MX2 using short hairpin RNAs increased HIV infection in IFNa-treated responsive cells but had no effect in the absence of the cytokine, which indicates that MX2 makes an important contribution to IFNa-induced restriction. Further experiments revealed that MX2 reduced the levels of 2-long terminal repeat circular DNA (which is a nuclear form of reverse-transcribed HIV DNA) and integrated viral DNA, which suggests that MX2 either decreases the stability of viral replication complexes or blocks their nuclear import.

Liu et al. also showed that MX2 reduces HIV DNA integration. To determine which viral proteins are targeted by MX2, the authors passaged HIV in MX2-expressing cells until MX2-resistant viruses evolved. The viruses acquired three mutations; one of these affected residue Ala88 in the viral capsid protein, which is known to interact with the cellular protein cyclophilin A (CYPA). Silencing CYPA increased HIV replication in MX2-expressing cells and immunoprecipitation experiments showed that CYPA and MX2 interact, which suggests that CYPA is crucial for targeting MX2 to the HIV capsid. Goujon et al. also found that viruses with mutated capsids had reduced sensitivity to MX2.

Taken together, the two studies show that MX2 is a novel IFNinduced inhibitor of HIV infection, which restricts HIV replication prior to integration. Interactions with CYPA and the viral capsid are important for this restriction, but the exact mechanism of inhibition is unknown. Interestingly, the closely related protein MX1 (also known as MXA) is a well-known inhibitor of many RNA and DNA viruses, including influenza virus. Both MX1 and MX2 contain a GTPase domain, and the GTPase function of MX1 is essential for the inhibition of influenza virus infection. By contrast, experiments by Goujon et al. provided no clear evidence for the involvement of the MX2 GTPase in HIV restriction. Thus, further work is needed to elucidate the mode of action of MX2 and to potentially harness it for new antiretroviral therapies.

Ursula Hofer

ORIGINAL RESEARCH PAPERS Goujon, C. et al. Human MX2 is an interferon-induced post-entry inhibitor of HIV-1 infection. Nature http://dx.doi. org/10.1038/nature12542 (2013) | Liu, Z. et al. The interferon-inducible MxB protein inhibits HIV-1 infection. Cell Host Microbe http://dx.doi. org/10.1016/j.chom.2013.08.015 